COMUNICAÇÃO CIENTÍFICA

\title{
MÉTODO NÃO DESTRUTIVO PARA DETERMINAÇÃO DA ÁREA FOLIAR DA VIDEIRA, CULTIVAR BRS-VIOLETA ${ }^{1}$
}

\author{
GUSTAVO MALAGI ${ }^{2}$, IDEMIR CITADIN ${ }^{3}$, SILVIA SCARIOT $^{2}$, LETÍCIA REIS $^{4}$
}

RESUMO- Este trabalho teve por objetivo avaliar as combinações entre o comprimento das nervuras secundárias e principal de folhas na estimativa da área foliar da videira cultivar BRS-Violeta. Realizou-se a coleta aleatória de 200 folhas intactas e completamente desenvolvidas, em uma área de cultivo experimental. Determinaram-se a área foliar real (AFR) e o comprimento das nervuras secundárias (esquerda - direita) e principal. Obtiveram-se três regressões com seus coeficientes de determinação para a identificação da relação mais precisa, considerando o comprimento da nervura principal (CNP), o somatório do comprimento das nervuras secundárias (SCNS) e o somatório entre o comprimento das nervuras secundárias e o comprimento da nervura principal (SCNSP), como variáveis independentes. A AFR foi considerada variável dependente nas três regressões. Observou-se que a relação entre AFR x SCNS proporcionou o maior coeficiente de determinação $(0,87)$. A área foliar estimada pela equação obtida pela relação AFR x SCNS garantiu precisão de $87 \%$, segundo a relação entre AFR x AFE (área foliar estimada). Portanto, conclui-se que a área foliar da videira cultivar BRS-Violeta pode ser estimada pela equação y $=0,2169$ (SCNS) ${ }^{2}+5,3642$ (SCNS) 34,725, com precisão satisfatória.

Termos para indexação: Vitis sp., nervura principal, nervura secundária, equação de área foliar.

\section{NON-DESTRUCTIVE METHOD FOR LEAF AREA DETERMINATION OF GRAPEVINE, BRS-VIOLETA CULTIVAR}

\begin{abstract}
The objective of this work was to evaluate combinations between lengths of secondary and main veins of leaves to estimate leaf area from grapevine, 'BRS-Violeta' cultivar. Random sampling was made from 200 entire and completely developed leaves, within an area of experimental cultivation. Actual leaf area (AFR) and the lengths of secondary (left-right) and main veins were determined. Three regressions were obtained with the coefficients of determination in order to identify the most accurate relation, considering main vein length (CNP), sum of secondary vein lengths (SCNS) and sum of secondary and main vein lengths (SCNSP), as independent variables. AFR was considered dependent variable in the three regressions. Relation between AFR x SCNS resulted in a higher coefficient of determination (0.87). Leaf area estimated by the equation obtained from AFR x SCNS relation was $87 \%$ accurate, according to AFR x AFE (estimated leaf area) relation. Therefore, leaf area from grapevine 'BRS-Violeta' cultivar can be estimated by the equation: $\mathrm{y}=0.2169(\mathrm{SCNS})^{2}+5.3642(\mathrm{SCNS})-34.725$, with adequate accuracy.
\end{abstract}

Index terms: Vitis sp., main vein, secondary vein, leaf area equation.

\footnotetext{
1(Trabalho 242-09). Recebido em: 19-10-2009. Aceito para publicação em: 22-09-2010.

${ }^{2}$ Eng. Agrônomo(a) mestrando(a) do Programa de Pós-Graduação em Agronomia da Universidade Tecnológica Federal do Paraná - UTFPR, Campus Pato Branco. Pato Branco-PR. Via do conhecimento, km 01. 85503-390. e-mails: malagi@agronomo.eng.br; silviascariotto@yahoo.com.br.

${ }^{3}$ Professor do Programa de Pós-Graduação em Agronomia da UTFPR, Campus Pato Branco. e-mail: idemir@utfpr.edu.br.

${ }^{4}$ Acadêmica do curso de Agronomia da UTFPR, Campus Pato Branco. e-mail: lereis04@hotmail.com
} 
O desenvolvimento da vitivinicultura no Brasil está baseado em grande parte nos resultados de pesquisa que buscam aprimorar as práticas de cultivo, visando a maiores produtividades e melhorando a qualidade da uva produzida. Os estudos ecofisiológicos, principalmente daqueles voltados na determinação da área foliar na cultura da videira, auxiliam na verificação da superfície fotossintética, o que permite a obtenção de indicadores importantes para o entendimento das respostas das plantas aos fatores ambientais ou de qualquer outro fator que influencie estas respostas (LOPES et al., 2004). Podemos mencionar como exemplos de indicadores a taxa transpiratória, a taxa de assimilação de $\mathrm{CO}_{2}$, a taxa de liberação de $\mathrm{O}_{2}$ e o vigor das plantas (CHAMPAGNOL, 1984), além do índice de área foliar.

A estimativa da área foliar pode ser obtida com o uso de métodos destrutivos e não destrutivos. Embora os métodos destrutivos sejam simples e precisos (SOMMER; LANG, 1994), estes apresentam o inconveniente de demandar muito tempo (LOPES et al., 2004), além de provocar a destruição da área foliar, o que impossibilita o acompanhamento do ciclo da cultura. A execução deste método ocorre normalmente por cópias em xerox e uso de planímetros (OLIVEIRA et al., 2002), ou mesmo através de aparelhos que quantificam a área foliar.

Os métodos não destrutivos são classificados em diretos e indiretos (MABROUK; CARBONNEAU, 1996). Os métodos não destrutivos diretos caracterizam-se por serem rápidos, precisos, e os resultados serem expressos imediatamente com o uso de equipamentos portáteis (BINDI et al., 1997; OLLAT et al., 1998; COHEN et al., 2000; DOBROWSKI et al., 2002), porém sua utilização é restrita devido ao alto custo de aquisição (OLIVEIRA et al., 2002) e há necessidade de manutenção e/ou ajustes frequentes nos equipamentos (OLLAT et al., 1998).

Os métodos não destrutivos indiretos são caracterizados por relações entre fatores, como área foliar e medições lineares realizadas em folhas e sarmentos, por exemplo. Estas relações são expressas em geral por equações de regressão que são utilizadas na estimativa da área foliar. Alguns trabalhos que descrevem a utilização de métodos não destrutivos em diversas culturas foram desenvolvidos (PEDRO JÚNIOR et al., 1986; RAMKHELAWAN; BRATHWAITE, 1990; GONÇALVES et al., 2002; OLIVEIRA et al., 2002; LOPES et al., 2004). No entanto, são necessários estudos específicos para cada cultivar e sistema de condução de plantas de videira para ajustar as equações de regressão, visando a reduzir o erro na quantificação da área foliar. Assim, o objetivo do trabalho foi avaliar o uso de combinações entre o comprimento das nervuras das folhas de videira, cultivar BRS-Violeta, na estimativa da área foliar.

O experimento foi instalado no campo experimental da Universidade Tecnológica Federal do Paraná, Câmpus Pato Branco, em solo pertencente à unidade de mapeamento LATOSSOLO VERMELHO distroférrico úmbrico, textura argilosa, álico, fase floresta subtropical perenifólia, com relevo ondulado (BHERING et al., 2008), em latitude $26^{\circ} 10^{\prime} 38^{\prime \prime}$ S e longitude $52^{\circ} 41$ ' 24 " W, a 764 metros de altitude. O clima local é do tipo Cfa (Classificação de Köeppen), subtropical com temperatura média no mês mais frio inferior a $18^{\circ} \mathrm{C}$ (mesotérmico) e temperatura média no mês mais quente acima de $22^{\circ} \mathrm{C}$, geadas pouco frequentes no inverno e tendência de concentração das chuvas nos meses de verão (MAACK, 1981), com precipitação anual média que varia de 2.000 a $2.500 \mathrm{~mm}$ (CAVIGLIONE et al., 2000).

Videiras da cultivar BRS-Violeta, enxertadas sobre o porta-enxerto Paulsen 1.103, foram plantadas em 2008 no espaçamento de 1 x 4 metros, na orientação norte-sul e conduzidas pelo sistema espaldeira, totalizando aproximadamente 3.600 plantas $\mathrm{ha}^{-1}$. Sete meses após o início das brotações e com os sarmentos desenvolvidos, 200 folhas completamente expandidas e sem danos aparentes foram coletadas, e suas áreas foliares mensuradas com o auxílio de um determinador de área foliar (marca LI-COR, modelo 3100) com valores expressos em $\mathrm{cm}^{2}$. Em seguida, cada folha foi identificada, e os comprimentos das nervuras principal e secundárias (esquerda e direita) foram mensurados por régua milimetrada com valores expressos em centímetros.

Com os resultados da área foliar real (AFR) e do comprimento das nervuras de cada folha, efetuou-se a análise dos pressupostos matemáticos seguida da análise de variância, considerando o delineamento inteiramente casualizado. Determinaramse três regressões, para a identificação da relação mais precisa, considerando o comprimento da nervura principal (CNP), o somatório do comprimento das nervuras secundárias (SCNS) e o somatório entre o comprimento das nervuras secundárias e o comprimento da nervura principal (SCNSP), como variáveis independentes. A AFR foi considerada variável dependente nas três regressões. A equação de regressão que apresentou o maior coeficiente de determinação foi utilizada na substituição da variável independente ' $\mathrm{x}$ ', por valores obtidos do somatório entre o comprimento das nervuras secundárias de cada folha. A área foliar estimada por esta equação foi relacionada com a área foliar real obtida pelo determinador de área foliar.

De acordo com a análise entre as três re- 
lações observadas, verificou-se que a relação entre 'AFR x CNP' (Figura 1) foi de 0,78, enquanto as relações entre 'AFR x SCNS' (Figura 2) e 'AFR x SCNSP' (Figura 3) apresentaram coeficiente de determinação de 0,87 . Considerando que quanto mais próximo de 1 for o coeficiente de determinação $\left(\mathrm{r}^{2}\right)$ maior é a precisão da equação de regressão na estimativa da área foliar, as relações SCNS e SCNSP foram consideradas as mais adequadas.

Porém, a estimativa da área foliar pode ser obtida de maneira mais simples com a utilização da equação (1) y = 0,2169 (SCNS) ${ }^{2}+5,3642$ (SCNS) 34,725 obtida pela situação da Figura 2. A área foliar estimada, obtida pela aplicação da equação 1 , garante uma proximidade satisfatória da AFR, uma vez que o coeficiente de determinação obtido pela relação entre estes dois fatores foi de 0,87 , conforme Figura 4. Oga e Fonseca (1994) e Regina et al. (2000) obtiveram coeficientes de determinação de 0,97 e 0,98 pela utilização de métodos não destrutivos que consideravam a relação entre comprimento e largura das folhas de pequizeiro e a soma do comprimento das nervuras secundárias das folhas de videira, respectivamente. Nascimento et al. (2002) verificaram uma alta relação entre a área foliar do meloeiro (Cucumis melo, L.) com o número e a largura de folhas.

Com base nos resultados obtidos, concluise que a estimativa da área foliar em folhas de videira, cultivar BRS-Violeta, pode ser efetuada por meio de método não destrutivo pela aplicação de equação quadrática com a utilização do somatório do comprimento das nervuras secundárias (SCNS).

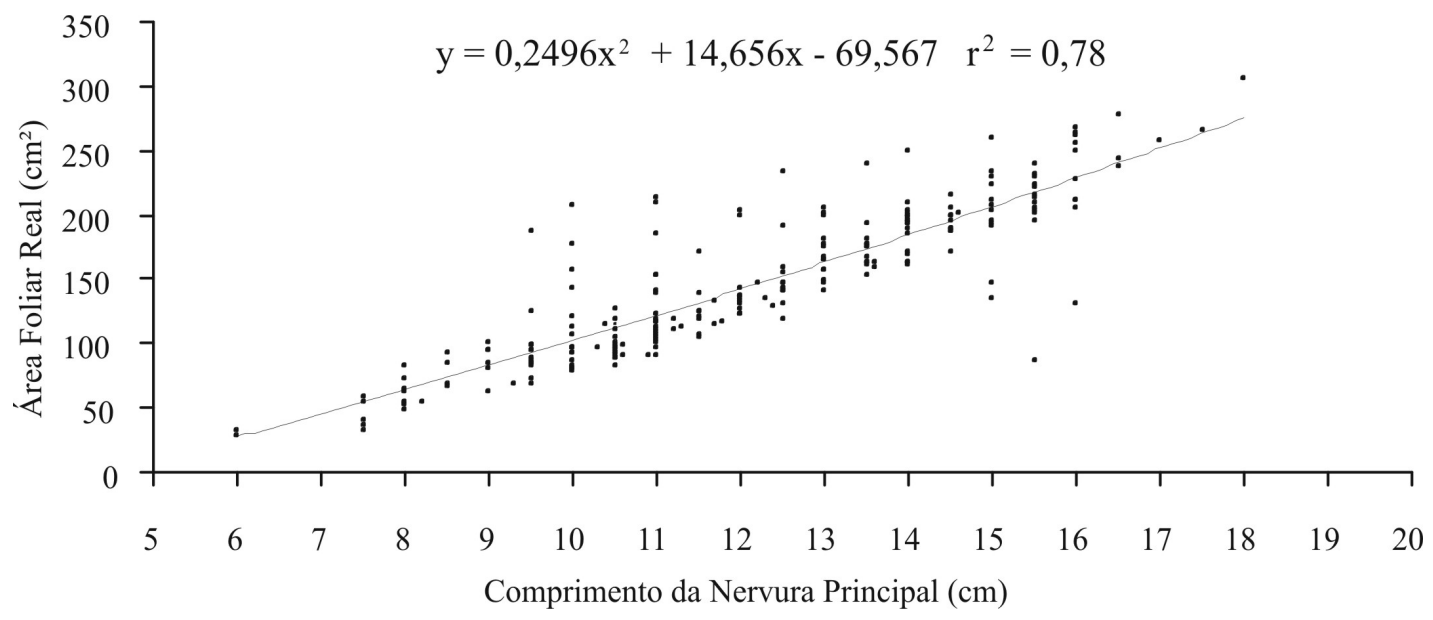

FIGURA 1 - Relação entre área foliar real $\left(\mathrm{cm}^{2}\right)$ e o comprimento $(\mathrm{cm})$ da nervura principal da folha de videira, cultivar BRS-Violeta.

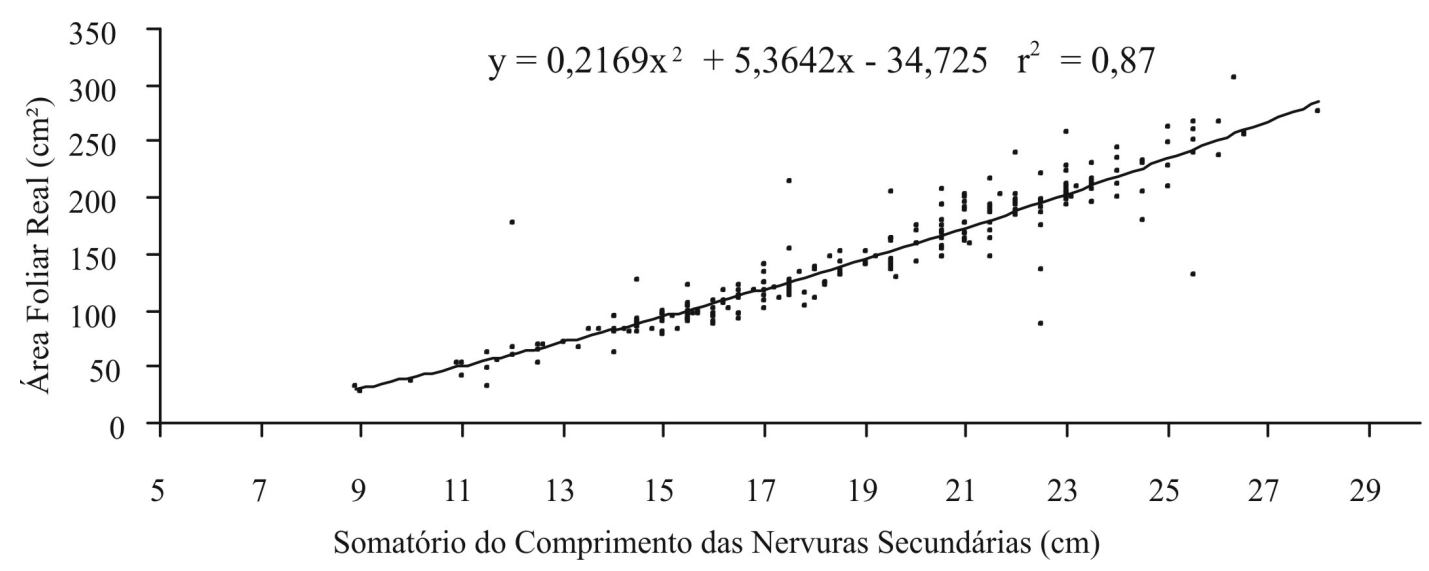

FIGURA 2 - Relação entre área foliar real $\left(\mathrm{cm}^{2}\right)$ e o somatório do comprimento $(\mathrm{cm})$ das nervuras secundárias da folha de videira, cultivar BRS-Violeta. 


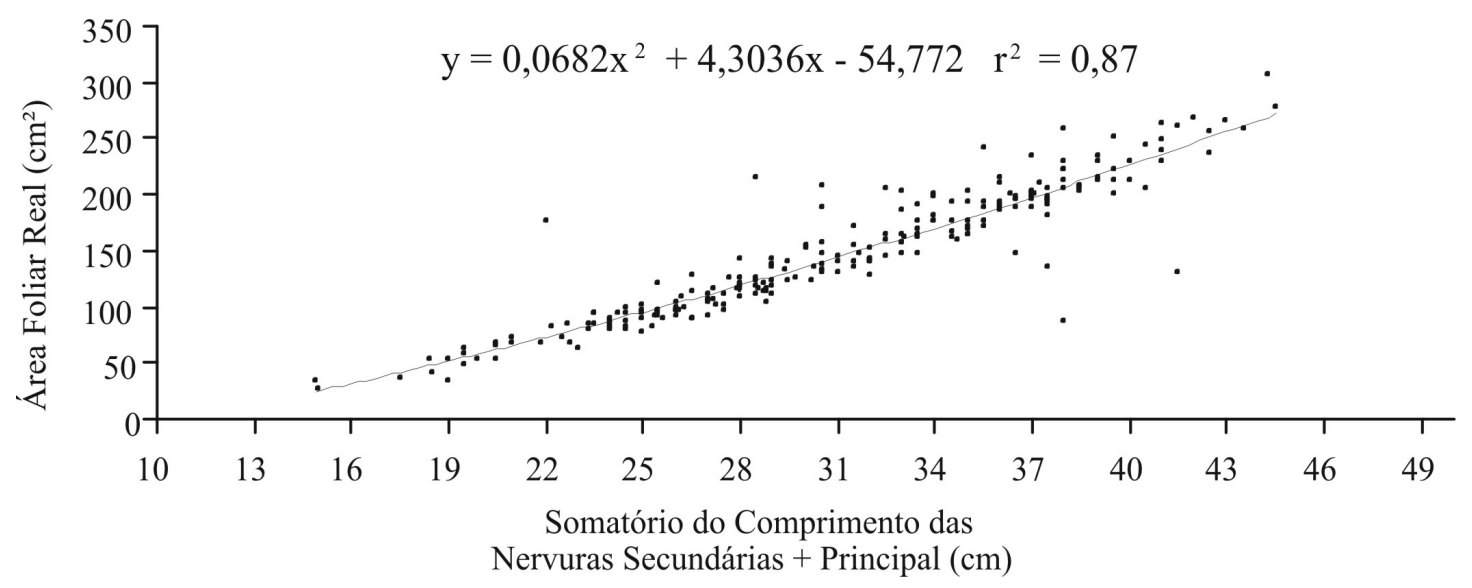

FIGURA 3 - Relação entre área foliar real $\left(\mathrm{cm}^{2}\right)$ e o somatório do comprimento $(\mathrm{cm})$ das nervuras secundárias e principal da folha de videira, cultivar BRS-Violeta.

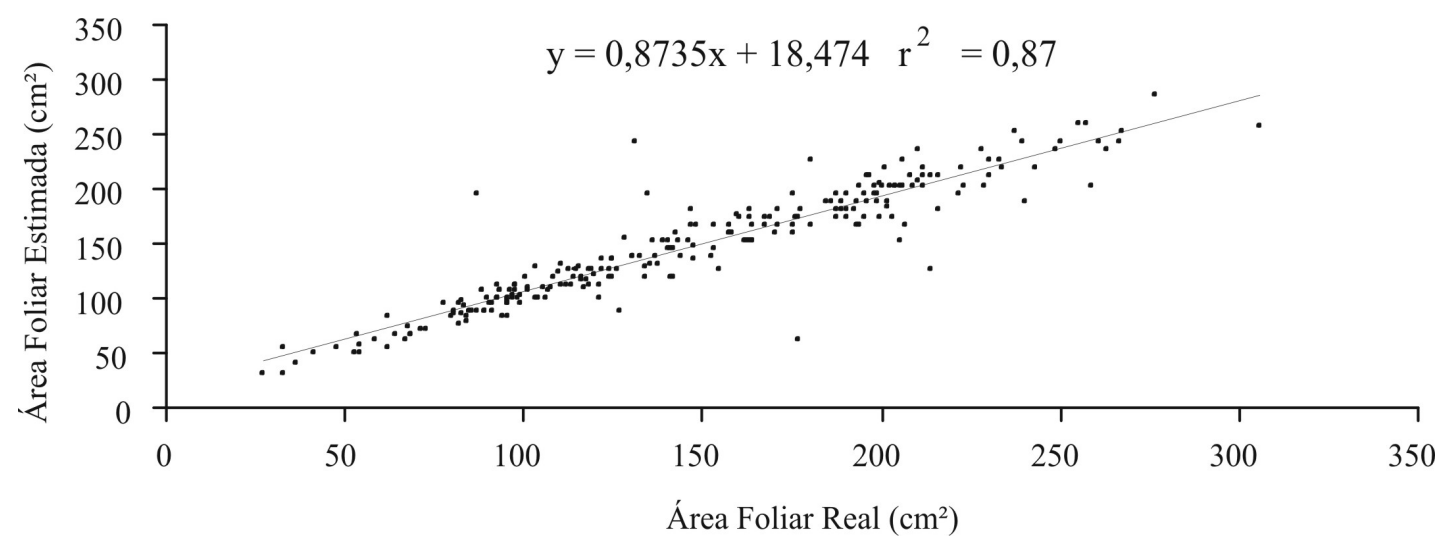

FIGURA 4 - Relação entre área foliar estimada $\left(\mathrm{cm}^{2}\right)$ e área foliar real $\left(\mathrm{cm}^{2}\right)$ pela equação y $=0,2169$ $(\mathrm{SCNS})^{2}+5,3642(\mathrm{SCNS})-34,725$, da videira cultivar BRS-Violeta. 


\section{REFERÊNCIAS}

BHERING, S.B.; SANTOS, H.G. dos; BOGNOLA, I.A.; CÚRCIO, G.R.; MANZATTO, C.V.; CARVALHO JUNIOR, W. de; CHAGAS, C da S.; ÁGLIO, M.L.D.; SOUZA, J.S. de. Mapa de solos do Estado do Paraná: legenda atualizada. Rio de Janeiro: EMBRAPA/IAPAR, 2008. 74p.

BINDI, M.; MIGLIETTA, F.; GOZZINI, B.; ORLANDINI, S.; SEGHI, L. A simple model for simulation of growth and development in grapewine (Vitis vinifera). Model description. Vitis, Siebeldingen, v.36, p.67-71, 1997.

CAVIGLIONE, J.H.; KIIHL, L.R.B.; CARAMORI, P.H.; OLIVEIRA, D. Cartas climáticas do Paraná. Londrina : IAPAR, 2000. CD-ROM.

CHAMPAGNOL, F. Elémentes de physiologie de la vigne et de viticulture general. Dehan, Montpellier: Dehan.,1984.

COHEN, S.; STRIEM, M.J.; BRUNER, M.; KLEIN, I. Grapevine leaf area índex evaluation by grap fraction inversion. Acta Horticulture, Wageningen, v.537, p.87-93, 2000.

DOBROWSKI, S.Z.; USTIN, S.L.; WOLPERT, J.A. Remote estimation of vine canopy density in vertically shoot-positioned vineyards: determining optimal vegetation indexes. Australian Journal of Grape and Wine, Adelaide, v.8, p.117-125, 2002.

GONÇALVES, C.A.A.; CHALFUN, N.N.J.; REGINA, M.A.; ALVARENGA, A.A.; SOUZA, M.T. de; ABRAHÃO, E. Estimativa de área foliar da videira (Vitis labrusca L. cv. Folha de figo) sobre diferentes porta-enxertos. Ciência e Agrotecnologia, Lavras, v.26, n.3, p.500-504, 2002.

LOPES, C.M.; ANDRADE, I.; PEDROSO, V.; MARTINS, S. Modelos empíricos para estimativa da área foliar da videira na casta Jaen. Ciência e Técnica Vitivinícola, Dois Portos, v.19, n.2, p.61-75, 2004.

MABROUK, H.; CARBONNEAU, A. Une méthode simple de determination de la foliare de la vigne (Vitis vinifera L.). Progrès Agricole et Viticole, Montpellier, v.113, n.18, p.392-398, 1996.
MAACK, V. Geografia física do Estado do Paraná. Rio de Janeiro: Livraria José Olympio, 1981. 442p.

NASCIMENTO, I.B.; FARIAS, C.H.A.; SILVA, M.C.C.; MEDEIROS, J.F.; ESPÍNOLA SOBRINHO, J.; NEGREIROS, M.Z. Estimativa da área foliar do meloeiro. Horticultura Brasileira, Brasília, v.20, n. 4, p.555-558, 2002.

OGA, F.M.; FONSECA, C.E.L. Um método rápido para estimar a área foliar em mudas de cagaiteira (Eugenia dysenterica D.C.). Pesquisa Agropecuária Brasileira, Brasília. v.29, n.4, p.571-577, 1994.

OLIVEIRA, M.N.S. de; LOPES, P.S.N.; MERCADANTE, M.O.; OLIVEIRA, G.L.; GUSMÃOR, E. Medição da área foliar do pequizeiro utilizando a soma da nervura principal dos folíolos. Unimontes Científica, Montes Claros, v.3, n.3, 2002.

OLLAT, N.; FERMAUD, M.; TANDONNET, J.P.; NEVEUX, M. Evaluation of an indirect method for leaf área index determination in the vineyard: combined effects of cultivar, year and training system. Vitis, Siebeldingen, v.37, p.73-78, 1998.

PEDRO JÚNIOR, M.J.; RIBEIRO, I.J.; MARTINS, F.P. Determinação da área foliar em videira cultivar 'Niágara Rosada'. Bragantia, Campinas. v.45, n.1, p.199-204, 1986.

RAMKHELAWAN, E.; BRATHWAITE, R.A.I. Leaf area estimation by non-destructive methods in sour orange (Citrus aurantium L.). Tropical Agriculture, Trinidad, v.67, n.3, p.203-206, 1990.

REGINA, M. de A.; PEREIRA, G. E.; CANÇADO, G. M. A.; RODRIGUES, D. J. Cálculo da área foliar em videira por método não destrutivo. Revista Brasileira de Fruticultura, Jaboticabal, v.22, n.3, p.310-313, 2000.

SOOMER, K.J.; LANG, A.R.G. Comparative analysis of two indirect methods of measuring LAI as applied to minimal and spur prunned grapes vines. Australian Journal Plant Physiologic, Melbourne, v.21, p.197-206, 1994. 